AIAA-2001-4425

\title{
FLIGHT SIMULATOR FIDELITY CONSIDERATIONS FOR TOTAL AIR LINE PILOT TRAINING AND EVALUATION*
}

\author{
Judith Bürki-Cohen \\ Volpe National Transportation Systems Center \\ U.S. Department of Transportation Research and Special Programs Administration \\ Cambridge, Massachusetts \\ Tiauw H. Go \\ Massachusetts Institute of Technology, Cambridge, Massachusetts \\ Thomas Longridge* \\ Federal Aviation Administration, Washington, D.C.
}

\begin{abstract}
This paper presents the FAA/Volpe Center's Flight Simulator Fidelity Research Program, which is part of the Federal Aviation Administration's effort to promote the effectiveness, availability and affordability of flight simulators. This initiative will become increasingly critical with the anticipated regulatory changes mandating the use of simulators in airline pilot training and evaluation, dramatically reduced pilot new-hire experience levels and growing operational complexity. Two research areas with high pay-off potential for this effort are radio communications and platform motion simulation. Initial results suggest that for fully effective training and evaluation of the cognitive and workload management skills associated with radio communications, significant improvements in radio communications realism are needed. Initial research on the training effectiveness of a fixed-base simulator with a wide field-of-view visual system compared to a like system having platform motion failed to find an operationally significant effect of motion. Follow-up work will examine whether this result was a function of the motion characteristics or the maneuvers tested. No changes in regulatory requirements can be expected without absolute confidence in the reliability and validity of the results, requiring considerable additional research in both areas.
\end{abstract}

\footnotetext{
* Portions of this work have been published as Ref. 1.

Engineering Psychologist, Member AIAA.

Postdoctoral Associate, Department of Aeronautics and Astronautics, Member AIAA.

* Manager, Advanced Qualification Program, AFS-230.

This paper is declared a work of the U.S. Government and is not subject to copyright protection in the United States.
}

\section{NOTATIONS}

AC Advisory Circular

AQP Advanced Qualification Program

ASP Advanced Simulation Plan

ASRS Aviation Safety Reporting System

ATC Air Traffic Control

CRM Crew Resource Management

DFS Deutsche Flugsicherung (German ATC)

DOF Degrees Of Freedom

FAA Federal Aviation Administration

FOV Field Of View

GATES Ground Air Traffic Environment System

I/E Instructor/Evaluator

IOE Initial Operating Experience

IRAS Interactive Real Time Audio System

JOINT Joint Operational Incidents Training

NAS National Airspace System

PF Pilot Flying

PNF Pilot Not Flying

NPRM Notice of Proposed Rulemaking

PTS Practical Test Standards

RTO Rejected Take-Off

SFAR Special Federal Aviation Regulation

SME Subject Matter Expert

TCAS Traffic Alert and Collision Avoidance System

$\mathrm{V}_{1} \quad$ Take-off decision speed; the minimum speed in the take-off, following a failure of the critical engine, at which the pilot can continue the take-off and achieve the required height above the take-off surface within the take-off distance.

$\mathrm{V}_{1}$ cut Engine failure at or above the take-off decision speed, $\mathrm{V}_{1}$, with continued take-off

$\mathrm{V}_{\mathrm{R}}$ cut Engine failure at or above the rotation speed, $\mathrm{V}_{\mathrm{R}}$, with continued take-off 


\section{INTRODUCTION}

This paper gives an overview of the Federal Aviation Administration's (FAA) initiative towards promoting the effectiveness, availability and affordability of flight simulators. It discusses simulator use in airline pilot training and evaluation as well as recent changes in training and evaluation needs and their implications for simulator fidelity requirements. It then explains the FAA's rationale and objectives for tasking the Volpe Center with a scientific review of simulator requirements that examines both the fidelity of the simulation of the environment in which the airplane operates and the fidelity of the airplane simulation itself. Finally, it presents the current focal points of the investigation, namely, the need $\square$ or lack of need $\square$ for realistically simulating radio communications and airplane motion. Findings to date and future plans are discussed.

\section{SIMULATOR USE IN AIRLINE PILOT TRAINING AND EVALUATION}

Simulators have been used in air-carrier pilot training and evaluation since the 1950s. These early simulations were effective for training and checking of procedures, systems knowledge, and navigation skills. Not until the introduction of the FAA's Advanced Simulation Plan (ASP), however, which established technical requirements for simulators, could stick and rudder skills be trained and evaluated in the simulator. Major airlines are currently not only conducting their recurrent training entirely in the simulator, but also their initial, transition, and upgrade training and certification. This is followed by carefully structured Initial Operating Experience (IOE) carrying passengers in the air, under supervision of a Check Airman.

The control and flexibility afforded by flight simulators has greatly enhanced pilot training as well as practically eliminated training accidents at major airlines that have access to the high-fidelity simulators required for total training and checking. Training accidents, however, continue to occur at smaller airlines that still conduct some of their training in the airplane. Also, the lack of access to high-fidelity simulators deprives the smaller airlines of some of the training opportunities provided by simulators, such as carefully constructed scenarios containing emergencies that require cognitive skills such as crew coordination, task management, and decision making in addition to motor flying skills. These training opportunities are particularly important in view of recent changes in the airline operational environment, effecting a shift in training and evaluation needs.

\section{TRAINING CHALLENGES IN TODAY'S OPERATIONAL ENVIRONMENT}

Managers of airline pilot training programs today are increasingly being faced with some unprecedented challenges. Of these, changing demographics in the available new-hire population is clearly the most prominent. Many of the U.S.'s largest airlines have found it necessary to dramatically decrease their entrylevel requirements in terms of flight hours and prior experience. This in turn has placed even greater pressure on smaller airlines, where entry levels have been lowered to bare minimums, while turnover among pilots leaving for positions with major airlines is at an all time high.

All of this is occurring in a backdrop of increased congestion within the National Airspace System (NAS), associated short-term strategies (such as Land and Hold Short) to manage capacity, the acquisition of newer aircraft with increasingly automated cockpit systems, the merging of airlines which may differ in their operating procedures and their corporate cultures, and near term plans for new ways of operating within the NAS, such as free flight and Air Traffic Control (ATC) data link.

These developments clearly have implications for the design and content of pilot training. Pilot training curricula, which were based on certain entry-level assumptions that are no longer valid, must be revised to incorporate training in areas either not previously required for airline new hires, or not previously addressed in depth. In some cases this will require a substantial increase in the footprints allocated to training and assessment of basic knowledge and handson flying skills. In all cases it will require that flight operations training include specific emphasis on building proficiency in the integration of cognitive and motor flying skills. While these needs are particularly pertinent to initial qualification curricula, they may also necessitate certain changes in recurrent training curricula, in order to assure that requisite proficiency is maintained, especially for rarely practiced skills, or those with very low prior experience histories.

Similarly, it becomes increasingly important for each airline to assure that pilot training for both newhire and existing pilot populations appropriately reflect its dynamically changing environment, whether that be in terms of aircraft systems, flight procedures, or corporate culture.

With regard to the regulatory environment pertinent to these developments, there is both good and bad news. On the positive side, the FAA has established a voluntary regulatory program for airlines that is well suited to meet the training challenges of today and 
tomorrow. The Advanced Qualification Program (AQP), which was established as Special Federal Aviation Regulation (SFAR) $58,{ }^{2}$ is specifically designed to assure that pilot training programs remain responsive to changing needs, and that the graduates of such programs not only possess the requisite knowledge and hands-on skills, but that in particular, they can demonstrate proficiency in the integration of cognitive and motor skills in operationally realistic scenarios that test both.

On the negative side, the traditional pilot training regulations, Subparts N \& O of Part 121 of the Code of Federal Regulations, which constitute the only alternative to $\mathrm{AQP}$, are sorely out of date relative to today's needs. The FAA is presently in the process of rewriting Subparts N \& O. A Notice of Proposed Rulemaking (NPRM) detailing proposed changes to traditional pilot training, testing, and checking requirements is anticipated at a future date.

AQP requires airlines to employ a systematic instructional-design process in determining the content of pilot training, testing, and checking, as well as in the training equipment allocation for those purposes to curricula. It specifically requires the use of entry-level analysis to assure that training content is appropriately matched to an airline's pilot population, and it mandates the implementation of a continuing quality control process for monitoring the effectiveness of curricula. While it allows flexibility in equipment selection, AQP is not ordinarily intended for use in curricula that employ the aircraft for training. Rather, AQPs typically employ a suite of training equipment, which includes flight training devices and full flight simulators.

The revised Subpart N \& O rules are expected to incorporate a similar philosophy. This is in contrast to the existing rules, which still permit training, testing, and checking to be conducted entirely in the aircraft. It can reasonably be expected, therefore, that within a few more years, all airline pilot training in the U.S. will require the use of full flight simulators for certain training, testing, and checking tasks, while allowing for the use of flight training devices for many but not all such tasks. It can also be expected that pilot training addressing both cognitive and motor skills in simulated scenarios that require both sets of skills will be a requirement for all curricula, both in AQP and otherwise.

A related regulatory development is the pending issuance of an NPRM on simulator qualification. Whereas presently U.S. simulator qualification procedures and standards are detailed only in an FAA Advisory Circular (AC), ${ }^{3}$ the proposed new rule is intended to establish a regulatory basis for those procedures and standards. If issued as a final rule, airlines and training centers that do not maintain their flight simulators in accordance with the procedures and standards on which basis the FAA originally qualified the equipment could be subject to FAA enforcement action.

\section{FAA/VOLPE FLIGHT SIMULATOR FIDELITY RESEARCH PROGRAM}

The net result of these developments is that ultimately all pilot training in the U.S. will be conducted in FAA qualified training equipment (full flight simulators, and, where permitted, flight training devices) rather than in aircraft. Airlines will be required by regulation to maintain the fidelity of such equipment in accordance with FAA qualification criteria. Furthermore, it can be expected that regardless of whether an airline is conducting pilot training under an AQP or under revised traditional rules, all curricula will require training in operationally realistic scenarios.

While these developments will be largely transparent to major airlines under AQP, they will be especially challenging to smaller airlines that do not presently employ simulators, or that have limited access to such equipment. Depending on the particular aircraft make, the availability of flight simulators may be problematic, and for some operators, cost could be prohibitive.

In anticipation of these regulatory changes, the FAA is committed to ensure that flight simulators effectively prepare pilots to carry passengers in the air. First, the simulator must faithfully represent both the cognitive and the motor challenges encountered in today's operational environment. Second, simulator fidelity requirements must be sufficient to ensure full transfer of behaviors and performance between airplane and simulators. In other words, for evaluation purposes, the skills, behaviors, and performance that a pilot demonstrates $\square$ or fails to demonstrate $\square$ in the air must be accurately reflected in the simulator. For training purposes, the skills, behaviors, and performance that a pilot acquires in the simulator must be available to him or her in the air.

To ensure access of all airlines large and small to the benefit of simulator training, however, the FAA must also ensure that the required means and level of fidelity for cue representation are necessary. That means that fidelity requirements must result in a demonstrable, operationally relevant transfer benefit, rather than being based on user preferences and technological availability alone.

The FAA has therefore tasked the Volpe Center with developing a scientific basis to assure that FAA requirements promote full transfer of pilot performance 
between simulator and airplane without unnecessarily driving up cost. A thorough review of the literature and systematic consultation of subject matter experts (SMEs) has shown that while the effectiveness of flight simulators for initial and recurrent training of airline pilots is well recognized, there are two areas that warrant further examination in light of the preceding discussion: the fidelity of the representation of radio communications and the requirement for platform motion cues during full flight simulations.

\section{REALISTIC RADIO COMMUNICATIONS SIMULATION FIDELITY REQUIREMENTS}

One of the widely recognized deficiencies in the current state of the art in full flight simulation is realistic radio communications. The simulation of such communications is typically accomplished by the pilot instructor/evaluator $(\mathrm{I} / \mathrm{E})$, who is also tasked with operating the simulator while observing trainee performance. This mode of simulation is a highly simplified representation of radio communications, and does not even begin to approach the challenges of hearing, acknowledging, and appropriately responding to radio communications within a real world environment. It follows that scenario-based training, testing, and checking that relies on such an unrepresentative means of simulation is not likely to be fully effective in developing the cognitive and workload management skills associated with radio communications. While this is obviously a deficiency of long standing, it may assume greater significance in light of the changing demographics of the pilot newhire population. It follows that research is needed to measure the impact of realistic radio communications on training effectiveness, and to make an assessment of whether there may be an affordable means of better simulating this function considering developing technology.

Volpe, in collaboration with NASA Ames, has therefore examined 1) airlines' current methods of simulating radio communications, 2) the effect of these practices on training and evaluation according to SMEs, literature, and reports on IOE, and 3) industry efforts to improve the current situation. A summary of this work follows. ${ }^{4}$

\section{Current Practices}

The findings summarized below are based on information collected from $29 \mathrm{I} / \mathrm{Es}$ from 14 AQP airlines, including seven major, one cargo, four regional, and two non-U.S. airlines. I/Es were queried about their simulation of different events, including ATC (tower, approach/departure, en route) and company communications (dispatch, ramp, maintenance, flight attendants) to own aircraft, ATC communications to and from other aircraft or ground vehicles (the so-called party line), as well as visual representation of other traffic.

A first finding was that the method of simulating radio communications is indeed almost exclusively $\mathrm{I} / \mathrm{E}$ role play, where the $\mathrm{I} / \mathrm{E}$ issues instructions and responds from his station directly behind the crew. This was found both for company and ATC radio communications.

With regard to communications to own airplane, all I/Es reported simulating ATC clearances in the terminal environment, and all but two provide communications en route. Fewer I/Es have time to role-play company communications, between 63 (ramp/gate) and 94 percent (dispatch).

I/Es were also queried about representation of other traffic. Only 59 percent indicated that their simulators provide some out-the-window view of traffic, mainly on the airport surface. Ten reported simulation of traffic via the Traffic Alert and Collision Avoidance System (TCAS). With regard to the party line, only 38 percent of all I/Es reported simulating any communications to and/or from other aircraft or vehicles, mainly on the airport surface.

In summary, it appears that although I/Es provide their best effort simulating radio communications, without additional support they have to focus their limited resources on role-playing communications to own airplane in the terminal environment.

\section{Instructor/Evaluator Opinions}

I/Es were asked to indicate their allocation of time and effort between running the simulation, simulating radio communications, instructing and observing. I/Es spend about 50 percent of their time and effort observing, twenty percent each role-playing radio communications and operating the simulator, and less than ten percent instructing.

Not surprisingly, I/Es rated their workload consistently higher for training and checking in the simulator than in the actual aircraft with real communications and no simulator to run. On the other hand, they feel that the communications workload of the pilots is significantly reduced. One I/E mentioned that even the manual workload of pilots is reduced, because "[p]ilots are not normally given a chart frequency, nor do they need to redial a new frequency to communicate."

I/Es stressed the importance of radio communications simulation for teaching such skills as (new) ATC procedures, Crew Resource Management (CRM) and situation awareness effectively. The overall 
importance of radio communications is perceived highest in the terminal environment. I/Es concern with simulating radio communications may have best been summarized by the I/E who stated: "Without communication simulation, when the pilot trainee finally arrives in the 'real world,' he must add another component... This new (additional) component can really complicate line flying."

\section{Literature Review}

Many of the I/E opinions found in the previous section are confirmed in the literature. ${ }^{4}$ FAA materials on AQP and CRM stress that coordination with ATC, company, and flight attendants is an integral part of line operations and that frequency monitoring is important for maintaining traffic and weather situation awareness. ${ }^{5,6}$

Incident and accident investigations highlight the importance of radio communications. The Flight Safety Foundation Approach and Landing Accident Reduction Task Force recommends that operators "[i]nclude training scenarios that allow crews to experience overload, task saturation, loss of situational awareness, out-of-control and too-far-behind-the aircraft-situations, and communications in stressful circumstances." Joint training should be held between pilots and air traffic controllers including scenarios that "promote mutual understanding of issues on both the flight deck and in the ATC environment, and foster improved communications during emergency situations. "7

There is much theoretical and experimental evidence that whole-task training in a fully loaded environment is superior to part-task training in an incomplete environment that may induce a false sense of operational simplicity akin to tunnel vision. ${ }^{8}$ Learning is an active process, and practice can lead to either activation or inhibition of cognitive pathways. ${ }^{9}$ If pilots are consistently exposed to an impoverished environment during training, they may end up unprepared for the "quick pace of an airline environment and [its] associated distractions," $\partial$ where their inexperience may prove fatal.

\section{Effect on Initial Operating Experience}

A search of the Aviation Safety Reporting System (ASRS) database from January 1993 to October 1999, resulting in 93 reports related to flight events during IOE, confirmed some of the concerns expressed by I/Es and the literature. ${ }^{10}$ Most of the errors reported involved altitude deviations or course deviations. Other multiple errors were landing or take-off without clearance,

\footnotetext{
${ }^{\partial}$ From an ASRS report
}

approach to or landing on the wrong runway or airport, runway incursions, and loss of communications.

Radio communications played a major role in 72 percent of the reports. Demanding, inadequate, or even erroneous ATC instructions spearheaded the factors leading to events deemed worthy of a report. Amended clearances requiring reprogramming of the automation or erroneous "expect" instructions were often cited. This was followed by inadequate CRM or task management related to radio communications, ATC interruptions including traffic calls and frequency congestion, stuck microphones blocking an entire frequency, or pilots stepping on an ongoing conversation. Next were radio-tuning problems and unfamiliar phraseology and accent, the latter usually in non-English speaking territory.

\section{Industry Initiatives}

Given all the evidence presented in the previous section, it is not surprising that both airlines and simulator industry are striving to improve radio communications realism. Only efforts known to have been used in airline training will be discussed. ${ }^{4,10}$

Among the most operationally realistic efforts is United Airlines' Interactive Real Time Audio System (IRAS). It is based on field recordings of actual ATC communications, including both communications to own and to and from other aircraft with controller voices dubbed with the individual instructors' voices. The system, however, is no longer operational due to high scenario-development, integration and instructortraining costs.

The Canadian simulator manufacturer CAE has developed the Ground Air Traffic Environment System (GATES) after a request from a foreign airline to provide a visual representation of traffic in the airport terminal environment. It soon became obvious that correlated and meaningful radio communications would have to be an essential component of such traffic representation. The I/E still provides all ATC communications to own airplane, however. Several domestic airlines and training facilities as well as foreign airlines and military are currently equipping their simulators with GATES.

Lufthansa and the German ATC organization Deutsche Flugsicherung (DFS) are collaborating on Joint Operational Incidents Training (JOINT). Up to eight Lufthansa simulators can be linked to two DFS ATC control sector simulators, resulting in highly effective recurrent training of both pilots and controllers. Each ATC simulator consists of a controller work station with a radar display showing the simulated airplanes flown by the flight simulator crews as well as 
other airplanes sharing the same airspace operated by a pseudo pilot sitting at a connected computer station.

\section{SIMULATOR MOTION FIDELITY REQUIREMENTS}

While all FAA qualified full flight simulators are required to have platform motion, a number of questions yet remain to be empirically addressed through appropriate research. Specifically, the question to be answered is whether the training conducted in a fixed-base simulator with a wide Field-Of-View (FOV) visual system produces a result equivalent to that obtained in a like system having platform motion cueing. An additional question from a regulatory perspective is whether proficiency checks conducted in a visually equipped fixed-base simulator provide an equivalent opportunity to verify the line-operational readiness of air-carrier pilots.

If the answers to these questions can reliably and validly be obtained, the FAA may be better able to determine what level of equipment should be required for initial or recurrent training programs in the future, and whether changes to future qualification criteria for such equipment are warranted. These decisions could significantly affect the cost and availability of flight training equipment in light of future regulatory plans, particularly for small operators.

\section{Subject Matter Expert Opinion}

The focus on simulator motion originated from a series of joint FAA-Industry symposia that were part of an FAA-sponsored review of the simulator requirements outlined in $\mathrm{AC} 120-40 \mathrm{~B} .^{3}$ This effort is summarized in Ref. 11. The intent was to systematically examine the most costly aspects of airplane simulation for potential cost-savings without loss in training effectiveness. A first symposium on the simulation of the aeromodel focusing on flight data collection and predictive modeling resulted in recommendations that would lower the cost of the simulator data package by $50 \% .^{12}$

Regarding simulator motion, the SMEs from industry, academia, and FAA participating in the discussions generally perceived that the absence of platform motion cueing in fixed-base devices is likely to have a detrimental effect on pilot control performance, particularly in maneuvers entailing sudden motion-onset cueing with limited visual reference. It was also noted, however, that there was no scientific evidence that training in a fixed-base device would lead to degraded control performance in the actual aircraft. This issue is especially pertinent in a device equipped with a wide FOV visual system, which can generate a relatively strong illusion of motion (vection). ${ }^{13}$

\section{Past Work}

An extensive literature review suggested that platform motion in the simulator might improve the acceptability of the simulator, at least when the pilots were aware of the motion manipulation. ${ }^{14,15}$ Motion also improved pilot performance and control behavior in the simulator, especially for disturbance tasks and tracking tasks of aircraft with low dynamic stability. ${ }^{16,17,18}$ Some of the benefits of platform motion have also been shown to transfer to a higher fidelity simulator. ${ }^{19}$ However, the benefits of platform motion have not been proven in the case of transfer of training to the airplane. ${ }^{20}$

Driven by the above findings and the FAA commitment to improve the availability and affordability of simulators, Volpe has been tasked to obtain objective data on simulator motion requirements. Questions to be answered include, but are not limited to, the following: Are there any flight tasks for which a measurable difference in simulator training effectiveness can be found with and without platform motion? What is the relationship in motion cueing effectiveness for a wide FOV visual display versus platform motion? Are existing platform motion qualification criteria optimal? Is there a relationship between pilot experience level and the effectiveness of platform motion for training?

\section{Results of First Study}

A first experiment was performed to answer some of these questions for a simulator that is routinely used for pilot training and evaluation. It used an "as-is" FAA qualified Level $\mathrm{C}$ simulator with a six Degree-OfFreedom (DOF) synergistic motion system and a wide FOV high-quality visual system, simulating a 30passenger turboprop airplane with twin wing-mounted engines. ${ }^{21,22}$ Deficiencies identified in a review of prior research designs were avoided by measuring both pilot stimulation and response, testing both maneuvers and pilots that are diagnostic of a need for motion, preventing pilot and instructor bias, and ensuring sufficient statistical power to capture operationally relevant effects. Experienced airline pilots were evaluated and trained in the simulator, half of them with and the other half without motion. Then the transfer of skills acquired by both groups during this training was tested in the simulator with the motion system turned on as a stand-in for the airplane (quasi-transfer). The test maneuvers selected were engine failures on take-off with either rejected take-off (RTO) or continued takeoff $\left(\mathrm{V}_{1 / \mathrm{R}}\right.$ cut $)$ in low visibility, which satisfied the 
criteria described in the literature as diagnostic for the detection of a motion requirement. These criteria included 1) closed loop, to allow for motion to be part of the control feedback loop to the pilot; 2) disturbance maneuver, to highlight an early alerting function of motion, ${ }^{18,23} 3$ ) high gain, to magnify any motion effects and to reduce the stability of the pilot/airplane control loop, $\left.{ }^{18} 4\right)$ high workload, to increase the need for redundant cues such as provided by motion, out-thewindow view, instruments and sound; and 5) short duration, to prevent pilots from adjusting to a lack of cues. Both subjective (I/E grades, questionnaires) and objective data recorded from the simulator were collected from the experiment.

The results of the study indicate that the motion provided by the test simulator did not, in an operationally significant way for the tasks tested, affect evaluation and training progress in the simulator, or transfer of training acquired in the simulator with or without motion to the simulator with motion. It also didn't consistently affect the Pilots' Flying (PF), Pilots' Not Flying (PNF), and I/Es' subjective perception of the PFs' performance, workload, and training, or of their own comfort in the simulator. Neither did it affect the acceptability of the simulator to the PF and the PNF.

Note, however, that because the current simulator qualification procedures do not provide a means to objectively assess the quality of the produced motion, ${ }^{24}$ the motion provided by the test simulator may not be typical of other FAA qualified Level C simulators. Specifically, the observed lack of lateral acceleration produced by the simulator shortly following the engine failure compared to the lateral acceleration from the aircraft mathematical model suggests that the simulator used in the study may not have provided sufficient motion stimulation to the pilot.

To validate and generalize the previous results, two follow-up studies are underway. One examines the typicality of the test simulator used in the previous experiment, by gathering data from other Level $\mathrm{C}$ and $\mathrm{D}$ simulators and then comparing these data to the test simulator. The second study will be similar to the previous one, while eliminating any possible causes of not having found an effect of motion.

\section{Comparison Study}

This effort is expected to provide information on whether the earlier results may apply to other FAA qualified simulators, at least for the maneuvers and pilot population tested. The main purpose of the comparison is to examine whether the level of lateral accelerations generated by the motion system and the equations of motion of the test simulator for the $V_{1}$ cut maneuver are typical. Data from nine level C and D simulators, representing turboprop or turbofan airplanes with twin wing-mounted engines, were obtained for comparison. Due to simulator time constraints, the requested data were obtained by running the maneuver using the automatic testing feature of the simulators.

There are several difficulties in performing the comparison. First of all, the simulators came from a range of manufacturers and as such the capability of accessing the data at various points in the simulation loop varied from one simulator to another. Therefore, not all the data requested were obtained from each simulator. Also, sometimes there was no way of telling whether values of certain variables recorded from the simulator computer had passed through some scaling. Second, the engine failure profiles used in the automatic testing vary greatly from one simulator to another, and these profiles can affect the resulting lateral acceleration. Thus, absolute comparison is not possible with the data gathered.

Within the above constraints, however, initial analysis of the data suggested that the level of lateral acceleration produced by the test simulator during $\mathrm{V}_{1}$ cut maneuvers is not atypical.

\section{Current Research}

As mentioned earlier, a study is currently underway to validate and generalize the previous results. As in the first study, the current effort is maximized for finding an effect of motion if there is one. An effort is made to eliminate any causes of not finding an effect of motion in the previous experiment. For example, the motion system of the test simulator will be tuned to provide the best possible performance within its operational envelope. Also, test subjects will fly different maneuvers considered to be diagnostic for detecting an effect of motion. Finally, the use of a different airplane simulation will automatically result in the use of a different pilot population.

This study will be performed in collaboration with the NASA Ames Research Center and will utilize its B747-400 simulator, which is an FAA qualified level D simulator. In collaboration with the manufacturer (CAE), some modifications on the motion system are performed to guarantee that the maximum capability of the motion actuators be utilized for flying the test maneuvers.

A between-subjects design is used for the experiment. Subjects will be divided into two groups, where one group will be evaluated and trained in the simulator with motion and the other in the simulator without motion. Both groups will then be tested for transfer of training in the simulator with motion as a stand-in for the real airplane (quasi-transfer). 
For the test maneuver, the selection criteria remain the same as in the previous study, with some relaxation of the short-duration criterion in favor of maneuvers that require a more complex correction by the pilot. The current plan is to combine a continued take-off with an engine failure and a high-workload landing task into one complete scenario. The candidates for the landing tasks are a sidestep landing with vertical gust disturbance on rollout and a precision instrument approach with varying crosswind disturbance. Dependent on the maneuver selection, the other settings in the simulator, such as airplane configuration and visibility, will be adjusted so that the motion stimulation and the pilot workload are maximized, while still complying with aviation regulations. Subjective (questionnaires, I/E grades) and objective (measurements, simulator recordings) data to assess motion stimulation of pilots, pilot performance, and pilot workload/behavior will be collected. Unlike the previous experiment, $\mathrm{I} / \mathrm{E}$ grades for the current experiment may have to be obtained offline due to cost and availability constraints.

Further preparation for the study and fine-tuning of the experiment design are currently underway. Results of this study will help determine whether the benefits of tighter motion standards, as currently considered, would justify the potential reduction in simulator availability due to an inevitable increase in acquisition, maintenance, as well as enforcement efforts.

\section{CONCLUSIONS}

Given the anticipated regulatory changes mandating the use of simulators in airline pilot training and evaluation, dramatically reduced pilot new-hire experience levels and growing operational complexity, the twin issues of training effectiveness and affordability of flight simulators for use by U.S. airlines will become increasingly critical. In that regard, two research areas with high pay-off potential are realistic radio communications and platform motion.

Initial FAA-sponsored research on the simulation of radio communications for U.S. airline pilot training strongly suggests that in order to be fully effective in developing the cognitive and workload management skills associated with radio communications, significant improvements are needed in the resources available to the pilot instructor for that purpose. The present practice of relying on IOE to compensate for the deficiencies in simulator resources for training radio communications skills may become increasingly inadequate in view of the changing demographics of the pilot new-hire population, as well as the anticipated future regulatory requirement for all airlines to conduct training in operationally realistic flight simulator scenarios. Although there have been a number of promising developments in this arena, considerable additional research is needed to reduce the cost and labor requirements associated with simulating radio communications in an operationally realistic fashion.

Initial research on the training effectiveness of a fixed-base simulator with a wide FOV visual system compared to a like system having platform motion failed to find an operationally significant effect of motion using FAA qualified equipment. Objective measurement of the motion characteristics of the FAA qualified simulator employed in the experiment, however, indicated that lateral acceleration stimulation was minimal. Subsequent comparisons with objective data acquired from other FAA qualified simulators indicated that the motion characteristics of the test simulator may be typical for the equipment routinely used for total airline pilot training and evaluation. A second training effectiveness study is planned using a test simulator tuned to provide the best possible platform motion performance and maneuvers selected to be especially diagnostic. However, considering the controversial nature of this issue, the safety implications, and the fact that no changes in regulatory requirements can be expected without absolute confidence in the reliability and validity of the results, it is likely that considerable additional research in this area will still be needed.

\section{ACKNOWLEDGMENTS}

This work was funded by the Office of the Chief Scientific and Technical Advisor for Human Factors of the Federal Aviation Administration, AAR-100. We are greatly indebted to Dr. Eleana Edens, the FAA program manager, for her excellent direction. The late Mr. Edward M. Boothe guided our steps throughout this project. We miss his knowledge, wisdom, insight, and friendship every day. The opinions expressed are those of the authors and not necessarily those of the Department of Transportation, the Federal Aviation Administration, or the U.S. Government.

\section{REFERENCES}

1. Longridge, T., Bürki-Cohen, J., Go, T. H., and Kendra, A. J., "Simulator Fidelity Considerations for Training and Evaluation of Today's Airline Pilots," Proceedings of the $11^{\text {th }}$ International Symposium on Aviation Psychology, The Ohio State University Press, Columbus, OH, March 2001. 
2. Federal Aviation Regulation, "Advanced Qualification Program,” 14 C.F.R., Part 121, SFAR 58, Washington, DC, 1990.

3. Federal Aviation Administration, "Airplane Simulator Qualification," Advisory Circular No. 120-40B, U.S. Department of Transportation, Washington, DC, 1991.

4. Bürki-Cohen, J., Kendra, A., Kanki, B., and Lee, A., "Realistic Radio Communication in Pilot Simulator Training," Report No. DOT/FAA/AR00/62, Washington, DC, 2000.

5. Federal Aviation Administration, Advanced Qualification Program, AFS-230, 2000, web site: http://www.faa.gov/avr/afs/aqphome.htm

6. Federal Aviation Administration, "Crew Resource Management Training," Advisory Circular No. 120-51C, U.S. Department of Transportation, Washington, DC, 1998.

7. Khatwa, R. and Helmreich, R., "Analysis of Critical Factors During Approach and Landing in Accidents and Normal Flight," Flight Safety Digest, Flight Safety Foundation, Alexandria, VA, 1999.

8. Fabiani, M., Buckley, J., Gratton, G., Coles, M., Donchin, E., and Logie, R., "The Training of Complex Task Performance," Acta Psychologica, 71, 1989, pp. 259-299.

9. Bransford, J. and Franks, J., "Towards a Framework for Understanding Learning," The Psychology of Learning and Motivation, 10, 1976, pp. 93-127.

10. Bürki-Cohen, J., and Kendra, A.J., "Air Traffic Control in Airline Pilot Simulator Training and Evaluation," Air Traffic Control Quarterly, accepted for publication pending revision.

11. Longridge, T., Ray, P., Boothe, E., and BürkiCohen, J., "Initiative Towards More Affordable Flight Simulators for U.S. Commuter Airline Training," Paper presented at the Royal Aeronautical Society Conference on Training-Lowering the Cost, Maintaining Fidelity, London, UK, May 1996.

12. Transcript ${ }^{\#}$ of the Joint FAA/Industry Symposium on Level B Airplane Simulator Aeromodel Validation Requirements, Washington Dulles Airport Hilton, March 13-14, 1996.

13. Transcript ${ }^{\#}$ of the Joint FAA/Industry Symposium on Level B Airplane Simulator Motion Requirements, Washington Dulles Airport Hilton, June 19-20, 1996.

\# Available in electronic format from Dr. Thomas Longridge, tel. (703) 661-0275
14. Reid, L. D. and Nahon, M. A., "Response of Airline Pilots to Variations in Flight Simulator Motion Algorithms," Journal of Aircraft, Vol. 25, No. 7, 1988, pp. 639-646.

15. Bussolari, S. R., Young, L. R., and Lee, A. T., "The Use of Vestibular Models for Design and Evaluation of Flight Simulator Motion," in AGARD CP-433, Paper presented at the Aerospace Medical Panel Symposium on Motion Cues in Flight Simulation and Simulator Induced Sickness, Brussels, Belgium, 1988.

16. Hosman, R. J. A. W. and van der Vaart, J. C., "Effects of Vestibular and Visual Motion Perception on Task Performance," Acta Psychologica, 48, 1981, pp. 271-287.

17. Hall, J. R., "Motion versus Visual Cues in Piloted Flight Simulation," in AGARD CP-249, Paper presented at the Flight Mechanics Panel Specialists Meeting on Piloted Aircraft Environment Simulation Techniques, Brussels, Belgium, 1978.

18. Hall, J. R., "The Need for Platform Motion in Modern Piloted Flight Training Simulators," Technical Memorandum FM 35, Royal Aerospace Establishment, London, UK, 1989.

19. Levison, W. H., "Effects of Whole-Body Motion Simulation on Flight Skill Development," Report AFOSR-TR-82-006, Air Force Office of Scientific Research, Washington, DC, 1981.

20. Waag, W. L., "Training Effectiveness of Visual and Motion Simulation," Report AFHRL-TR-7972, Air Force Human Resource Laboratory, Brooks Air Force Base, Texas, 1981.

21. Go, T. H., Bürki-Cohen, J., and Soja, N. N., "The Effect of Simulator Motion on Pilot Training and Evaluation," AIAA Paper 2000-4296, Proceedings of AIAA Modeling and Simulation Technologies Conference, Denver, CO, August 2000.

22. Bürki-Cohen, J., Boothe, E.M., Soja, N.N., DiSario, R.D., Go, T., and Longridge, T., "Simulator Fidelity-The Effect of Platform Motion," Proceedings of the Royal Aeronautical Society conference on Flight Simulation $\square$ The Next Decade, London, UK, May 2000.

23. Gundry, J., "Man and Motion Cues," Paper presented at the Third Flight Simulation Symposium, London, April, 1976.

24. Lahiri, A., "Significance of a Qualitative Handling Assessment Approach Towards Motion System Requirements for Flight Simulators," Proceedings of the Royal Aeronautical Society conference on Flight Simulation-A Decade of Regulatory Change: Has the Situation Changed for the Better? London, UK, 2000. 